\title{
Generalized Linear Differential Systems
}

\author{
WILLIAM T. REID
}

Communicated by W. Feller

1. Introduction. The generalized differential system treated here is equivalent to a type of linear vector Riemann-Stieltjes integral equation, and includes as special instances certain differential systems with "interface" conditions at a finite number of points; examples of such a system may also occur in a simple integral non-parametric variational problem as the accessory system along an extremizing arc with a finite number of corners (see, for example, REID [8]). Also, the real scalar generalized second order differential equations occurring in the works of Sz.-NAGY ([12] and [11; pp. 247-254]) and FELLER ([2]; see also earlier papers of FELLER referred to in [2]) are particular cases of the general system considered here.

Section 2 is concerned with an existence theorem and related basic properties of solutions. For self-adjoint systems it is shown in Section 3 that there exist criteria of oscillation and non-oscillation that are direct generalizations of known criteria for the usual self-adjoint differential system, while Section 4 deals with the extension to such systems of the oscillation, separation, and comparison theorems occurring in the generalization of the classical Sturmian theory due to Monse ([6], [7; Chs. III, IV]); Section 5 is concerned with the extension of the results of REID [10] on principal solutions. Section 6 is devoted to a system of second order difference equations that occurs as a special instance of the general system of Section 2. Finally, in Section 7 there is considered a more general system, which under a suitable non-singular linear transformation is equivalent to a system of the form treated in the earlier portion of the paper.

Matrix notation is used throughout; in particular, matrices of one column are termed vectors, and for a vector $\left(y_{\alpha}\right)(\alpha=1, \cdots, n)$ the norm $|y|$ is given by $\left(\left|y_{1}\right|^{2}+\cdots+\left|y_{n}\right|^{2}\right)^{\frac{1}{2}}$. The $n \times n$ identity matrix is denoted by $E_{n}$, or merely $E$ when there is no ambiguity, and 0 is used indiscriminately for the zero matrix of any dimensions; the conjugate transpose of a matrix $M$ is designated by $M^{*}$. If $M$ is an $n \times n$ matrix the symbol $|M|$ is used for the supremum of $|M y|$ on

This research was supported by the United States Air Force through the Air Force Office of Scientific Research of the Air Research and Development Command, under Contract No. AF18(603)-86. Reproduction in whole or in part is permitted for any purpose of the United States Government. 
the unit sphere $|y|=1$. The relations $M \geqq N(M>N)$ are used to signify that $M$ and $N$ are hermitian matrices of the same dimensions and $M-N$ is a nonnegative (positive) definite matrix. If an hermitian matrix $M(x), a \leqq x \leqq b$, is such that $M\left(x_{1}\right)-M\left(x_{2}\right) \geqq 0(\leqq 0)$ for $a \leqq x_{1}<x_{2} \leqq b$, then $M(x)$ is termed non-increasing (non-decreasing) on the interval $[a, b]: a \leqq x \leqq b$.

If a matrix $M(x)$ is a.c. (absolutely continuous) on an interval $[a, b]$, then $M^{\prime}(x)$ signifies the matrix of derivatives at values for which these derivatives exist and the zero matrix elsewhere. Correspondingly, if $M(x)$ is (Lebesgue) integrable on $[a, b]$ then $\int_{a}^{b} M(x) d x$ denotes the matrix of integrals of the respective elements of $M(x)$. If $M(x) \equiv\left\|M_{\alpha \beta}(x)\right\|(\alpha=1, \cdots, n ; \beta=1, \cdots, r)$ is an $n \times r$ matrix of b.v. (bounded variation) on $[a, b]$, while $A(x) \equiv\left\|A_{i \alpha}(x)\right\|$ $(i=1, \cdots, m ; \alpha=1, \cdots, n)$ and $B(x) \equiv\left\|B_{\beta i}(x)\right\|(\beta=1, \cdots$, $r ; j=1, \cdots, s)$ are continuous on this interval, then $\int_{a}^{b} A(x)[d M(x)] B(x)$ denotes the $m \times s$ matrix with elements given by the Riemann-Stieltjes integrals

$$
\sum_{\alpha=1}^{n} \sum_{\beta=1}^{r} \int_{a}^{b} A_{i \alpha}(x) B_{\beta j}(x) d M_{\alpha \beta}(x) .
$$

The symbols $\int_{a}^{b}[d M(x)] B(x)$ and $\int_{a}^{b} A(x)[d M(x)]$ denote $\int_{a}^{b} E_{n}[d M(x)] B(x)$ and $\int_{a}^{b} A(x)[d M(x)] E_{r}$, respectively. The utilized properties of such matrix Riemann-Stieltjes integrals are elementary in nature, and are direct consequences of well known theorems on scalar Riemann-Stieltjes integrals (see, for example, WidDer [13; Ch. 1]).

2. An existence theorem. In this and the major portion of the following section we shall be concerned with a fixed non-degenerate compact interval $J: a \leqq x \leqq b$ on the real line, and $R(x), M(x)$ are $n \times n$ matrices satisfying the following hypothesis:

(H) $R(x)$ is non-singular with $R(x)$ and $R^{-1}(x)$ (Lebesgue) integrable on $J$, and $M(x)$ is of b.v. on this interval.

By a solution $u(x), v(x)$ of the generalized vector differential system

$$
R(x) u^{\prime}(x)=v(x), \quad d v(x)=[d M(x)] u(x)
$$

on the interval $J$ will be meant an a.c. $u(x)$ and an associated $v(x)$ of b.v. on $J$ such that

$$
v(x)=\int_{a}^{x}[d M(t)] u(t)+v(a), \quad x \varepsilon J
$$

and $R(x) u^{\prime}(x)=v(x)$ a.e. (almost everywhere) on $J$. It is to be remarked that the validity of (2.2) is clearly equivalent to the existence of an $x_{0} \varepsilon J$ and a constant vector $v_{0}$ such that

$$
v(x)=\int_{x_{0}}^{x}[d M(t)] u(t)+v_{0}, \quad x \varepsilon J .
$$


Equally well, one might write

$$
d\left[R(x) u^{\prime}(x)\right]-[d M(x)] u(x)=0
$$

instead of (2.1); however, as many of our results involve explicitly both the vector function $u(x)$ and the associated $v(x)$ we shall consistently refer to the system (2.1).

The most common example of (2.1) is that in which $M(x)=\int_{x_{0}}^{x} P(t) d t$, where $P(x)$ is integrable on $J$, so that $u(x), v(x)$ is a solution of (2.1) if and only if this pair is a solution of the differential system

$$
u^{\prime}(x)=R^{-1}(x) v(x), \quad v^{\prime}(x)=P(x) u(x) ;
$$

that is, both $u(x)$ and $v(x)$ are a.c. and (2.3) holds a.e. on $J$. Another frequently occurring case is $M(x)=S(x)+\int_{x_{0}}^{x} P(t) d t$, where $P(x)$ is integrable and the elements of $S(x)$ are step functions on $J$; that is, there is a finite sequence of points $a=x_{0}<x_{1}<\cdots<x_{k}=b$ such that $S(x)$ is constant on each open interval $\left(x_{i-1}, x_{j}\right)(j=1, \cdots, k)$. In this case $u(x), v(x)$ is a solution of $(2.1)$ if and only if this pair is a solution of (2.3) on each subinterval $\left(x_{i-1}, x_{i}\right)$, while the right- and left-hand limits of these vector functions satisfy the "interface" conditions

$$
\begin{gathered}
u\left(x_{j}\right)=u\left(x_{i}^{-}\right)=u\left(x_{i}^{+}\right), \\
v\left(x_{i}\right)-v\left(x_{i}^{-}\right)=\left[S\left(x_{i}\right)-S\left(x_{i}^{-}\right)\right] u\left(x_{i}\right), \\
v\left(x_{i}^{+}\right)-v\left(x_{i}\right)=\left[S\left(x_{i}^{+}\right)-S\left(x_{i}\right)\right] u\left(x_{i}\right) \quad(j=0,1, \cdots, k),
\end{gathered}
$$

with the understanding that at $x_{0}=a$ and $x_{k}=b$ only the appropriate one-sided relations of $\left(2.3^{\prime}\right)$ are to be considered.

The basic result of this section is the following existence theorem.

Theorem 2.1. If $R(x), M(x)$ satisfy hypothesis $(\mathrm{H})$, then for a given $x_{0} \varepsilon J$ and constant vectors $u_{0}, v_{0}$ there is a unique solution of (2.1) satisfying the initial conditions

$$
u\left(x_{0}\right)=u_{0}, \quad v\left(x_{0}\right)=v_{0} .
$$

Clearly $u(x), v(x)$ is a solution of (2.1) satisfying (2.4) if and only if $u(x)$ is a solution of the integral equation

$$
u(x)=g(x)+\int_{x_{0}}^{x} R^{-i}(t)\left\{\int_{x_{0}}^{t}[d M(s)] u(s)\right\} d t, \quad x \varepsilon J,
$$

where $g(x)$ is the vector function

$$
g(x)=u_{0}+\left(\int_{x_{0}}^{x} R^{-1}(t) d t\right) v_{0}
$$

and $v(x)$ is given by $\left(2.2^{\prime}\right)$.

By the familiar method of iterations one may show that (2.5) has a unique 
solution for given $x_{0}, u_{0}, v_{0}$. Let

$$
\begin{aligned}
u_{0}(x) & \equiv 0, \\
u_{m+1}(x) & =g(x)+\int_{x_{0}}^{x} R^{-1}(t)\left(\int_{x_{0}}^{t}[d M(s)] u_{m}(s)\right) d t \quad(m=0,1,2, \cdots),
\end{aligned}
$$

and denote by $h(x)$ a monotone non-decreasing function such that

$$
|M(t)-M(s)| \leqq h(t)-h(s) \text { for } t, s \varepsilon J, \quad s<t .
$$

Such a function $h(x)$ may be specified in a variety of ways. One of the most natural is to define for an arbitrary subinterval $J_{1}: a_{1} \leqq x \leqq b_{1}$ of $J$ the function $h\left(J_{1}\right)$ as the supremum of $\sum_{j=1}^{k}\left|M\left(s_{i}\right)-M\left(s_{j-1}\right)\right|$ on the class of partitions $a_{1}=s_{0}<s_{1}<\cdots<s_{k}=b_{1}$ of $J_{1}$, and set

$$
\begin{gathered}
h\left(x_{0}\right)=0, \quad h(x)=h\left(\left[x_{0}, x\right]\right) \text { for } x>x_{0}, \\
h(x)=-h\left[\left(x, x_{0}\right]\right) \text { for } x<x_{0} .
\end{gathered}
$$

If $h_{J}=h(b)-h(a)$, and if for an arbitrary vector function $u(x)$ continuous on $J$ the symbol Max $\left(|u| ; x_{0}, t\right)$ denotes the maximum of $|u(x)|$ on the subinterval of $J$ with endpoints $x=x_{0}$ and $x=t$, then

$$
\left|\int_{x_{0}}^{t}[d M(s)] u(s)\right| \leqq h_{J} \operatorname{Max}\left(|u| ; x_{0}, t\right) .
$$

If $r(x)=\left|R^{-1}(x)\right|$ and $g_{J}$ is a constant such that $|g(x)| \leqq g_{J}$ on $J$, then by induction it follows that for $x \varepsilon J$

$$
\left|u_{m+1}(x)-u_{m}(x)\right| \leqq \frac{g_{J}}{m !}\left(h_{J}\left|\int_{x_{0}}^{x} r(s) d s\right|\right)^{m} \quad(m=0,1, \cdots) .
$$

Indeed, the definitive property of $g_{J}$ implies (2.10) for $m=0$, and if (2.10) is valid for $m=k(k=0,1, \cdots)$, then for $t \varepsilon J$

$$
\begin{aligned}
\left|\int_{x_{0}}^{t}[d M(s)]\left(u_{k+1}(s)-u_{k}(s)\right)\right| & \leqq h_{J} \operatorname{Max}\left(\left|u_{k+1}-u_{k}\right| ; x_{0}, t\right) \\
& \leqq \frac{g_{J} h_{J}}{k !}\left(h_{J}\left|\int_{x_{0}}^{t} r(s) d s\right|\right)^{k},
\end{aligned}
$$

and (2.10) for $m=k+1$ is an immediate consequence of the inequality

$$
\left|u_{k+2}(x)-u_{k+1}(x)\right| \leqq\left|\int_{x_{0}}^{x} r(t)\right| \int_{x_{0}}^{t}[d M(s)]\left(u_{k+1}(s)-u_{k}(s)\right)|d t| .
$$

Consequently, on $J$ the sequence $\left\{u_{m}(x)\right\}$ converges uniformly to a continuous limit vector function $u(x)$. From the recursive relation of (2.7) it follows readily that $u(x)$ is a solution of (2.5) on $J$. The uniqueness property follows in the usual fashion from the fact that if $u(x)$ is a solution of (2.5) with $g(x) \equiv 0$ and $|u(x)| \leqq u_{J}$ on $J$, then by induction 


$$
|u(x)| \leqq \frac{u_{J}}{m !}\left(h_{J}\left|\int_{x_{0}}^{x} r(s) d s\right|\right)^{m} \quad(m=0,1, \cdots)
$$

for $x \varepsilon J$, and hence $u(x) \equiv 0$ on $J$.

If $u=u\left(x, x_{0}, u_{0}, v_{0}\right), v=v\left(x, x_{0}, u_{0}, v_{0}\right)$ is the solution of (2.1) satisfying (2.4), then these functions do not possess the continuity properties with respect to the initial values $x_{0}, u_{0}, v_{0}$ that persist for the special case of the differential system (2.3). However, since in the above proof the constant $g_{J}$ may be chosen uniformly for $x_{0}, u_{0}, v_{0}$ on a set

$$
D_{k}: \quad x_{0} \varepsilon J, \quad\left|u_{0}\right| \leqq k, \quad\left|v_{0}\right| \leqq k \quad(k>0),
$$

from the above existence proof one may deduce readily the following result, which will be used subsequently in Section 4 .

Corollary. For a given $k>0$, there exists a $c_{k}>0$ such that

$\left|u\left(x, x_{0}, u_{0}, v_{0}\right)\right| \leqq c_{k}, \quad\left|v\left(x, x_{0}, u_{0}, v_{0}\right)\right| \leqq c_{k} \quad$ for $\quad\left(x_{0}, u_{0}, v_{0}\right) \varepsilon D_{k}, \quad x \varepsilon J ;$ moreover, uniformly for $x_{0} \varepsilon J$ the vector function $u\left(x, x_{0}, u_{0}, v_{0}\right)$ is continuous in $\left(x, u_{0}, v_{0}\right)$ on $D_{k}$, and uniformly for $x, x_{0} \varepsilon J$ the vector function $v\left(x, x_{0}, u_{0}, v_{0}\right)$ is continuous in $\left(u_{0}, v_{0}\right)$ on $\left|u_{0}\right| \leqq k,\left|v_{0}\right| \leqq k$.

Also for subsequent use we shall state a continuity property for solutions of a system

$$
R(x, \lambda) u^{\prime}(x)=v(x), \quad d v(x)=[d M(x, \lambda)] u(x),
$$

where $R(x, \lambda), M(x, \lambda)$ satisfy the following hypothesis.

$\left(\mathrm{H}_{\lambda}\right)$ For fixed $\lambda$ on $\lambda_{1} \leqq \lambda \leqq \lambda_{2}$ the $n \times n$ matrices $R(x, \lambda), M(x, \lambda)$ satisfy hypothesis $(\mathrm{H})$, and, moreover,

(a) there is a $\phi(x)$ integrable on $J: a \leqq x \leqq b$ such that $\left|R^{-1}(x, \lambda)\right| \leqq \phi(x)$ for $x \varepsilon J, \lambda_{1} \leqq \lambda \leqq \lambda_{2}$, while for fixed $\lambda_{0}$ on $\lambda_{1} \leqq \lambda_{0} \leqq \lambda_{2}$ we have for $x$ a.e. on $J$ that $R^{-1}(x, \lambda) \rightarrow R^{-1}\left(x, \lambda_{0}\right)$ as $\lambda \rightarrow \lambda_{0}$;

(b) if $\lambda_{1} \leqq \lambda_{0} \leqq \lambda_{2}$ then the total variation of $M(x, \lambda)-M\left(x, \lambda_{0}\right)$ on $J$ tends to zero as $\lambda \rightarrow \lambda_{0}$.

Whenever $\left(\mathrm{H}_{\lambda}\right)$ is satisfied the constant $g_{J}$ in the proof of Theorem 2.1 may be chosen uniformly for $\left(x_{0}, u_{0}, v_{0}\right) \varepsilon D_{k}(k>0)$ and $\lambda_{1} \leqq \lambda \leqq \lambda_{2}$; moreover, $h_{J}$ may be chosen uniformly for $\lambda$ on $\lambda_{1} \leqq \lambda \leqq \lambda_{2}$. Let $u=u\left(x, x_{0}, u_{0}, v_{0}, \lambda\right)$, $v=v\left(x, x_{0}, u_{0}, v_{0}, \lambda\right)$ denote the solution of (2.12) satisfying (2.4) and $u_{m}=$ $u_{m}\left(x, x_{0}, u_{0}, v_{0}, \lambda\right)$ the approximations (2.7). For fixed $x_{0} \varepsilon J$ the sequence $\left\{u_{m}\left(x, x_{0}, u_{0}, v_{0}, \lambda\right)\right\}$ converges uniformly on a set

$$
\lambda_{1} \leqq \lambda \leqq \lambda_{2}, \quad x \varepsilon J, \quad\left|u_{0}\right| \leqq k, \quad\left|v_{0}\right| \leqq k \quad(k>0),
$$

and by induction one may prove that each $u_{m}\left(x, x_{0}, u_{0}, v_{0}, \lambda\right)$ is continuous in $\left(x, u_{0}, v_{0}, \lambda\right)$ on $(2.13)$, so that one has the following result.

Theorem 2.2. If hypothesis $\left(\mathrm{H}_{\lambda}\right)$ is satisfied and $u=u\left(x, x_{0}, u_{0}, v_{0}, \lambda\right), v=$ $v\left(x, x_{0}, u_{0}, v_{0}, \lambda\right)$ is the solution of (2.12) satisfying (2.4), then for fixed $x_{0} \varepsilon J$ 
the vector function $u\left(x, x_{0}, u_{0}, v_{0}, \lambda\right)$ is continuous in $\left(x, u_{0}, v_{0}, \lambda\right)$ on $x \varepsilon J$, $\left|u_{0}\right|<\infty,\left|v_{0}\right|<\infty, \lambda_{1} \leqq \lambda \leqq \lambda_{2}$, and for fixed $x, x_{0} \varepsilon J$ the vector function $v\left(x, x_{0}, u_{0}, v_{0}, \lambda\right)$ is continuous in $\left(u_{0}, v_{0}, \lambda\right)$ on $\left|u_{0}\right|<\infty,\left|v_{0}\right|<\infty$, $\lambda_{1} \leqq \lambda \leqq \lambda_{2}$.

In connection with the establishment of these additional properties of solutions, the above type of existence proof appears preferable to that employed by FELLER $[2 ;$ pp. 466,467$]$ for the case of a real scalar equation of the form (2.5).

The theory of solutions of (2.1) on $J: a \leqq x \leqq b$ is intimately connected with the functional $I\left(\eta_{1}, \eta_{2} ; a, b\right)$ defined as

$$
I\left(\eta_{1}, \eta_{2} ; a, b\right)=\int_{a}^{b} \eta_{1}^{\prime *}(x) R(x) \eta_{2}^{\prime}(x) d x+\int_{a}^{b} \eta_{1}^{*}(x)[d M(x)] \eta_{2}(x)
$$

for brevity, $I(\eta ; a, b)$ will be written for $I(\eta, \eta ; a, b)$. If $u(x), v(x)$ is a solution of (2.1) then $u(x)$ is a.c. and $R(x) u^{\prime}(x)$ is essentially bounded on $J$. On the other hand, if $u(x)$ is a.c. and $R(x) u^{\prime}(x)$ is essentially bounded on $J$, then, for $\eta(x)$ an arbitrary a.c. $n$-dimensional vector function on $J$ and $w(x)=\int_{a}^{x}[d M(s)] u(s)$, we have

$$
I(\eta, u ; a, b)=\left.\eta^{*}(x) w(x)\right|_{a} ^{b}+\int_{a}^{b} \eta^{*}(x)\left[R(x) u^{\prime}(x)-w(x)\right] d x .
$$

From the fundamental lemma of the calculus of variations it follows that $I(\eta, u ; a, b)=0$ for arbitrary a.c. $\eta(x)$ satisfying $\eta(a)=0=\eta(b)$ if and only if there is a constant vector $v_{0}$ such that $R(x) u^{\prime}(x)-w(x)=v_{0}$ a.e. on $J$, which is equivalent to the condition that $u(x), v(x)=w(x)+v_{0}$ is a solution of (2.1) on $J$. Thus we have established the following result.

Lemma 2.1. For a given vector function $u(x)$ there is an associated $v(x)$ such that $u(x), v(x)$ is a solution of (2.1) on $a \leqq x \leqq b$ if and only if on this interval $u(x)$ is a.c., $R(x) u^{\prime}(x)$ is essentially bounded, and $I(\eta, u ; a, b)=0$ for arbitrary a. $c$. n-dimensional vector functions $\eta(x)$ satisfying $\eta(a)=0=\eta(b)$.

In particular, if $u(x), v(x)$ is a solution of (2.1) on $a \leqq x \leqq b$ and $u\left(x_{1}\right)=$ $0=u\left(x_{2}\right)$, where $a \leqq x_{1}<x_{2} \leqq b$, then from (2.15) it follows that $I\left(u ; x_{1}, x_{2}\right)=0$.

Now if $R=R_{0}(x), M=M_{0}(x)$ satisfy hypothesis (H) the matrices $R=R_{0}^{*}(x)$, $M=M_{0}^{*}(x)$ also satisfy this hypothesis. In particular, if $y(x), z(x)$ is a solution of the system adjoint to (2.1),

$$
R^{*}(x) y^{\prime}(x)=z(x), \quad d z(x)=\left[d M^{*}(x)\right] y(x)
$$

on $J: a \leqq x \leqq b$, and $u(x), v(x)$ is a solution of (2.1) on this interval, then for $a<s \leqq b$ it follows by the argument that led to $(2.15)$ that $I(y, u ; a, s)=$ $y^{*}(s) v(s)-y^{*}(a) v(a)$. Interchanging the roles of $y(x)$ and $u(x)$ it follows that $I(y, u ; a, s)=z^{*}(s) u(s)-z^{*}(a) u(a)$, so that there is a constant $c$ such that $y^{*}(x) v(x)-z^{*}(x) u(x) \equiv c$ on $J$. 
3. Self-adjoint systems. This section will be devoted to an equation (2.1) that is self-adjoint, that is, $R(x) \equiv R^{*}(x)$ and $M(x) \equiv M^{*}(x)$ on $J: a \leqq x \leqq b$. For brevity, let $\Lambda$ denote the totality of $n$-rowed matrices $U(x)$ which are a.c. on $J$ and for which there is a corresponding matrix $V(x)$ of b.v. such that $R(x) U^{\prime}(x)=V(x)$ a.e. on this interval. If $U(x) \varepsilon \Lambda$ and $V(x)$ satisfies with $U(x)$ the definitive property of $\Lambda$, we write $U(x) \varepsilon \Lambda: V(x)$. In particular, if $U_{j}(x) \varepsilon \Lambda(j=1,2)$, then

$$
I\left(U_{1}, U_{2} ; a, b\right) \equiv \int_{a}^{b} U_{1}^{\prime *}(x) R(x) U_{2}^{\prime}(x) d x+\int_{a}^{b} U_{1}^{*}(x)[d M(x)] U_{2}(x)
$$

exists and is finite; as in the case of $(2.14), I(U ; a, b)$ is written instead of $I(U, U ; a, b)$. We shall denote by $\Gamma(a, b)$ the totality of $n$-dimensional vector functions $\eta(x)$ which are a.c. and such that $I(\eta ; a, b)$ exists and is finite, and by $\Gamma_{0}(a, b)$ the subclass of $\Gamma(a, b)$ satisfying $\eta(a)=0=\eta(b)$. The following properties may be established readily.

Lemma 3.1. If $U_{j}(x) \varepsilon \Lambda: V_{i}(x)(j=1,2)$, then for $a<t \leqq b$

$$
\begin{aligned}
I\left(U_{1}, U_{2} ; a, t\right)=\left.U_{1}^{*} V_{2}\right|_{a} ^{t}-\int_{a}^{t} U_{1}^{*}(x)\left[d\left(V_{2}(x)-\int_{a}^{x}[d M(s)] U_{2}(s)\right)\right], \\
\left.\left(U_{1}^{*} V_{2}-V_{1}^{*} U_{2}\right)\right|_{a} ^{t}=\int_{a}^{t} U_{1}^{*}(x)\left[d\left(V_{2}(x)-\int_{a}^{x}[d M(s)] U_{2}(s)\right)\right] \\
-\left(\int_{a}^{t} U_{2}^{*}(x)\left[d\left(V_{1}(x)-\int_{a}^{x}[d M(s)] U_{1}(s)\right)\right]\right)^{*} .
\end{aligned}
$$

In particular, if $U(x) \varepsilon \Lambda: V(x)$ and $U^{*}(x) V(x)-V^{*}(x) U(x)$ is constant on $a \leqq x \leqq b$, then the matrix

$$
\int_{a}^{t} U^{*}(x)\left[d\left(V(x)-\int_{a}^{x}[d M(s)] U(s)\right)\right], \quad a \leqq t \leqq b,
$$

is hermitian.

Lemma 3.2. If $U(x)$ is an $n \times r$ matrix with $U(x) \varepsilon \Lambda: V(x)$ and $\xi(x)$ is an a.c. $r$-dimensional vector function such that $\eta(x)=U(x) \xi(x) \varepsilon \Gamma(a, b)$, then

$$
\begin{aligned}
I(\eta ; a, b)= & \left.\eta^{*} V \xi\right|_{a} ^{b}+\int_{a}^{b}\left[\xi^{*} U^{*} R U \xi^{\prime}-\xi^{*}\left(U^{*} V-V^{*} U\right) \xi^{\prime}\right] d x \\
& -\int_{a}^{b} \xi^{*}(t)\left[d\left(\int_{a}^{t} U^{*}(x)\left[d\left(V(x)-\int_{a}^{x}[d M(s)] U(s)\right)\right]\right)\right] \xi(t) .
\end{aligned}
$$

If $U(x) \equiv\left\|U_{\alpha j}(x)\right\|, V(x) \equiv\left\|V_{\alpha i}(x)\right\|(\alpha=1, \cdots, n ; j=1, \cdots, r)$ are $n \times r$ matrices, for typographical simplicity the symbol $(U(x) ; V(x))$ is used for the $2 n \times r$ matrix whose $j^{\text {th }}$ column has elements $U_{1 i}(x), \cdots, U_{n i}(x), V_{1 j}(x)$, $\cdots, V_{n j}(x)$. In particular, $(U(x) ; V(x))$ is a solution of

$$
R(x) U^{\prime}(x)=V(x), \quad d V(x)=[d M(x)] U(x),
$$


if the individual column vectors of $(U(x) ; V(x))$ are solutions of (2.1). If $\left(U_{1}(x) ; V_{1}(x)\right)$ and $\left(U_{2}(x) ; V_{2}(x)\right)$ are solutions of (3.6), then from (3.3) it follows that the matrix $U_{1}^{*}(x) V_{2}(x)-V_{1}^{*}(x) U_{2}(x)$ is a constant. This matrix will be denoted by $\left\{U_{1}, U_{2}\right\}$; it is to be remarked that there is no ambiguity in this notation, since if $(U(x) ; V(x))$ and $\left(U(x) ; V_{0}(x)\right)$ are solutions of (3.6) then $V(x) \equiv V_{0}(x)$. As for differential systems (REID [9]), if $u_{j}(x), v_{j}(x)(j=1,2)$ are solutions of (2.1) with $\left\{u_{1}, u_{2}\right\} \equiv 0$ these solutions are termed (mutually) conjoined. If $(U(x) ; V(x))$ is a $2 n \times r$ matrix whose column vectors are linearly independent mutually conjoined solutions of $(2.1)$, then $(U(x) ; V(x))$ is a matrix of conjoined solutions which has constant rank $r$, and the set of all solutions linearly dependent on these columns vectors is called a conjoined family of dimension $r$. As for ordinary differential systems (see REID [9; Lemma 2.3]), the maximum dimension of a conjoined family is $n$, and a given conjoined family of dimension $r<n$ is contained in a conjoined family of dimension $n$. In particular, if $(U(x) ; V(x))$ is a $2 n \times n$ matrix of conjoined solutions of rank $n$, then $(U(x) ; V(x))$ is called a base of the corresponding conjoined family $F$ of solutions, and $F$ is said to have at $x=x_{0}$ a focal point of order $k$ if $U\left(x_{0}\right)$ is of rank $n-k$.

Two points $x_{1}, x_{2}$ of $J$ are said to be (mutually) conjugate if there exists a solution $u(x), v(x)$ of $(2.1)$ with $u\left(x_{1}\right)=0=u\left(x_{2}\right)$ and $u(x) \equiv 0$ on $\left[x_{1}, x_{2}\right]$. Moreover, if there exist exactly $k$ linearly independent solutions $u_{i}(x), v_{j}(x)$ $(j=1, \cdots, k)$ of $(2.1)$ with $u_{i}\left(x_{1}\right)=0=u_{j}\left(x_{2}\right)$, then $x_{2}$ is said to be a conjugate point of $x_{1}$ with order $k$.

The system (2.1) is termed non-oscillatory on a given interval provided no two distinct points of this interval are conjugate. Corresponding to the notation of REID [9], the symbol $H_{+}(a, b)$ will denote the condition that $I(\eta ; a, b)$ is positive definite on $\Gamma_{0}(a, b)$, that is, $I(\eta ; a, b) \geqq 0$ for $\eta(x) \varepsilon \Gamma_{0}(a, b)$ and the equality sign holds only if $\eta(x) \equiv 0$ on $J$. Corresponding to Theorem 2.1 of REID [9], for (2.1) one has the following basic result.

Theorem 3.1. A necessary and sufficient condition that $H_{+}(a, b)$ hold is that $R(x)>0$ a.e. on $J: a \leqq x \leqq b$, together with one of the following conditions:

(i) The equation (2.1) is non-oscillatory on $J$.

(ii) If $\left(U_{1}(x) ; V_{1}(x)\right)$ is a solution of (3.6) with $U_{1}(a)=0$ and $V_{1}(a)$ nonsingular, then $U_{1}(x)$ is non-singular on $a<x \leqq b$.

(iii) If $\left(U_{2}(x) ; V_{2}(x)\right)$ is a solution of (3.6) with $U_{2}(b)=0$ and $V_{2}(b)$ nonsingular, then $U_{2}(x)$ is non-singular on $a \leqq x<b$.

(iv) There exists a matrix $(U(x) ; V(x))$ of conjoined solutions of (3.6) of rank $n$, with $U(x)$ non-singular on $J$.

(v) There exists an $n \times n$ matrix $U(x)$ with $U(x) \varepsilon \Lambda: V(x), U^{*}(x) V(x)-$ $V^{*}(x) U(x) \equiv 0, U(x)$ non-singular on $J$, and such that the corresponding hermitian matrix (3.4) is non-increasing on $J$.

In the above theorem only condition (v) differs formally from the corresponding condition of Theorem 2.1 of REID [9]. Moreover, the results of the above 
theorem may be established in the same manner as the corresponding results of the cited theorem of the earlier paper on differential systems; the one deviation of more than formal character is the use of relation (3.5) to show that $H_{+}(a, b)$ is a consequence of $(\mathrm{v})$ and the condition that $R(x)>0$ a.e. on $J$. In this connection, it is to be noted that if $N(x)$ is an hermitian matrix of b.v. on $J: a \leqq x \leqq b$, then $N(x)$ is constant (non-increasing) on this interval if and only if for all $c$, $d$ with $a \leqq c<d \leqq b$ the integral $\int_{c}^{d} \xi^{*}(x)[d N(x)] \xi(x)$ is zero (nonpositive) for arbitrary $n$-dimensional vector functions $\xi(x)$ continuous on $[c, d]$. Also, if $N_{1}(x)$ is a non-singular continuous matrix on $J$ and $N_{2}(x)$ is of b.v. on this interval, then $\int_{a}^{t} N_{1}(x)\left[d N_{2}(x)\right]$ is constant for a $\leqq \leqq b$ if and only if $N_{2}(x)$ is constant on $J$. These facts are of use in establishing for (2.1) results analogous to conditions iv $_{\mathbf{R}}$ and $\mathrm{v}_{\mathbf{R}}$ of REID [9] involving Riccati differential equations. Indeed, if $U(x)$ is non-singular and $U(x) \varepsilon \Lambda: V(x)$, then $W(x)=V(x) U^{-1}(x)$ is of b.v. on $J$ and $U^{*} V-V^{*} U \equiv U^{*}\left(W-W^{*}\right) U$, so that $U^{*} V-V^{*} U \equiv 0$ if and only if $W$ is hermitian on $J$; conversely, if $W(x)$ is of b.v. on $J$ and $U(x)$ is a non-singular a.c. matrix satisfying $R(x) U^{\prime}(x)=W(x) U(x)$ a.e. on this interval, then $V(x)=W(x) U(x)$ is of b.v. and $U(x) \varepsilon \Lambda: V(x)$. Moreover, if $U(x)$ is non-singular on $J, U(x) \varepsilon \Lambda: V(x)$, and $W(x)=V(x) U^{-1}(x)$, then, for $a \leqq c<d \leqq b, \xi(x)$ continuous on $[c, d]$ and $\xi_{1}(x)=U(x) \xi(x)$, we have

$$
\begin{aligned}
\int_{c}^{d} \xi^{*}(t)\left[d \left\{\int_{a}^{t} U^{*}(x)\right.\right. & {\left.\left.\left[d\left\{V(x)-\int_{a}^{x}[d M(s)] U(s)\right\}\right]\right\}\right] \xi(t) } \\
& =\int_{c}^{d} \xi_{1}^{*}(t)\left[d\left\{\int_{a}^{t}[d V(s)] U^{-1}(s)-M(t)\right\}\right] \xi_{1}(t), \\
& =\int_{c}^{d} \xi_{1}^{*}(t)\left[d\left(W(t)-M(t)-\int_{a}^{t} V(s)\left[d U^{-1}(s)\right]\right)\right] \xi_{1}(t), \\
& =\int_{c}^{d} \xi_{1}^{*}(t)\left[d\left(W(t)-M(t)+\int_{a}^{t} W(s) R^{-1}(s) W(s) d s\right)\right] \xi_{1}(t)
\end{aligned}
$$

the last relation follows from the preceding relation since $U^{-1}(x)$ is a.c. and $\left[U^{-1}(x)\right]^{\prime}=-U^{-1}(x) R^{-1}(x) W(x)$ a.e. on $J$. Consequently, for such $U(x)$ the matrix (3.4) is constant or non-increasing on $J$ if and only if the corresponding hermitian matrix

$$
W(x)-M(x)+\int_{a}^{x} W(s) R^{-1}(s) W(s) d s
$$

is constant or non-increasing on this interval, and in view of the comments at the beginning of this section the conditions (iv) and (v) of the above theorem are equivalent to the following respective conditions:

$\left(\mathrm{iv}^{\prime}\right)$ There exists an $n \times n$ hermitian matrix $W(x)$ of $b$. $v$. on $J$ such that (3.7) is constant on this interval.

$\left(\mathrm{v}^{\prime}\right)$ There exists an $n \times n$ hermitian matrix $W(x)$ of b.v. on $J$ such that (3.7) is non-increasing on this interval. 
Corresponding to the results of Section 4 of REID [9], for the system (2.1) one may derive specific criteria of oscillation and non-oscillation on an arbitrary interval $J_{0}$ of the real line. For brevity, we shall list here only the following three specific criteria.

(a) Suppose that $R(x) \equiv E$ and $M(x)$ is an hermitian matrix on a given interval $J_{0}$ which is of b.v. on each compact subinterval $J$ of $J_{0}$. If there exists a constant hermitian matrix $M_{0}$ and a value $x_{0} \varepsilon J_{0}$ such that

$$
M(x)+4 \int_{x_{0}}^{x}\left(M(s)-M_{0}\right)^{2} d s
$$

is non-increasing on $J_{0}$, then (2.1) is non-oscillatory on $J_{0}$.

(b) Suppose that $R(x)$ and $M(x)$ are hermitian matrices satisfying hypothesis (H) on arbitrary compact subintervals $J$ of $J_{0}: a<x<\infty, R(x)>0$ a.e. on $J_{0}$, and $M(x)$ is non-increasing on $J_{0}$. If there exists a non-null constant vector $\xi$ such that $\xi^{*} R(x) \xi=0(x)$ and $\xi^{*} M(x) \xi \rightarrow-\infty$ as $x \rightarrow \infty$, then (2.1) is oscillatory on each subinterval $a_{0} \leqq x<\infty$ of $J_{0}$.

(c) Suppose that $R(x)$ and $M(x)$ are hermitian matrices satisfying $(\mathrm{H})$ on a compact interval $J: a \leqq x \leqq b$ and $R(x)>0$ a.e. on this interval. If there exists a non-decreasing scalar function $m(x)$ on $J$ such that $m(x) E+M(x)$ is nondecreasing on this interval, and the hermitian matrix

$$
4\left(\int_{a}^{b} R^{-1}(x) d x\right)^{-1}-[m(b)-m(a)] E
$$

is non-negative definite, then (2.1) is non-oscillatory on $J$.

Criterion (a), which is an extension of conditions $\left(a^{\prime}\right)$ and $\left(a^{\prime \prime}-i\right)$ given on pp. 746-747 of REID [9], follows immediately from the above criterion $\left(\mathrm{v}^{\prime}\right)$ with $W(x)=2\left(M(x)-M_{0}\right)$.

In order to establish criterion (b), for $a_{0} \leqq x<\infty$ a subinterval of $J_{0}$ and $a_{0}+1<c<b$, let $\eta(x)=\left(x-a_{0}\right) \xi$ on $\left[a_{0}, a_{0}+1\right], \eta(x) \equiv \xi$ on $\left[a_{0}+1, c\right]$ and $\eta(x)=[(b-x) /(b-c)] \xi$ on $[c, b]$, where $\xi$ is a constant vector with the stated property in (b). As $M(x)$ is non-increasing on $J_{0}$, we have

$$
\begin{aligned}
I(\eta ; a, b) \leqq I\left(\eta ; a_{0}, a_{0}+1\right)+\xi^{*}[M(c)- & \left.M\left(a_{0}+1\right)\right] \xi \\
& +(b-c)^{-2} \int_{c}^{b} \xi^{*} R(x) \xi d x .
\end{aligned}
$$

Since $\xi^{*} R(x) \xi=O(x)$ as $x \rightarrow \infty$ the last integral of (3.8) is bounded uniformly for large $c, b$, and as $\xi^{*} M(x) \xi \rightarrow-\infty$ as $x \rightarrow \infty$ it follows that $I(\eta ; a, b)<0$ for $c$ sufficiently large. In view of Theorem 3.1 it then follows that (2.1) is oscillatory on $\left[a_{0}, b\right]$ for $b$ sufficiently large. Criterion (b) is an extension to systems (2.1) of a special case of Theorem 4.1 of REID [9].

Finally, criterion (c) is a direct extension of Theorem 4.2 of REID [9], and is a ready consequence of Theorem 2.3 of [9] and the remark that if $\eta(x) \varepsilon \Gamma_{0}(a, b)$ with $\eta(x) \equiv 0$ and the maximum value of $|\eta(x)|$ on $J$ is attained at $x=c$, then 


$$
\int_{a}^{b} \eta^{*}(x)[d M(x)] \eta(x) \geqq-[m(b)-m(a)]|\eta(c)|^{2} .
$$

As in the case of a system of ordinary differential equations (see, for example, Theorem 2.2 of $\operatorname{REID}_{\text {[ }}$ [9]), if (2.1) is self-adjoint and $H_{+}(a, b)$ holds on an interval $J: a \leqq x \leqq b$, then each solution $u(x)$ of (2.1) affords a proper absolute minimum to $I(\eta ; a, b)$ in the class of $\eta(x) \varepsilon \Gamma(a, b)$ and satisfying $\eta(a)=u(a), \eta(b)=u(b)$.

4. Sturmian theorems for self-adjoint systems. Throughout this section we shall consider a self-adjoint system (2.1) whose coefficient matrices satisfy $(\mathrm{H})$ on arbitrary compact subintervals $J$ of a given interval $J_{0}$, and $R(x)>0$ a.e. on $J_{0}$. In order to show that such a system admits Sturmian oscillation and comparison theorems of the same type as have been obtained for self-adjoint differential systems with real coefficients (see Morse [6] and [7; Chs. III, IV]; also BirkHoFF \& Hestenes [1] and Hestenes [5]), one needs to establish for (2.1) certain results whose usual proofs for differential systems depend upon properties of solutions that do not persist for the general system (2.1).

Lemma 4.1. If $J: a \leqq x \leqq b$ is a compact subinterval of $J_{0}$, then there exists $a \delta>0$ such that if $x_{1}, x_{2} \varepsilon J$ and $\left|x_{1}-x_{2}\right|<\delta$, then $x_{1}$ and $x_{2}$ are not conjugate. Moreover, if $F$ is a conjoined family of solutions of (2.1) with base $(U(x) ; V(x))$, then on any open subinterval of $J$ of length not exceeding $\delta$ there are at most $n$ focal points of $F$, each focal point being counted a number of times equal to its order; in particular, the focal points of a conjoined family $F$ of solutions are isolated.

The first conclusion of the lemma is an immediate consequence of Theorem 3.1 and the fact that the Corollary to Theorem 2.1 implies the existence of a $\delta>0$ such that if $x_{0} \varepsilon J$ and $\left(U_{0}(x) ; V_{0}(x)\right)$ is the solution of (3.6) determined by $U_{0}\left(x_{0}\right)=E, V_{0}\left(x_{0}\right)=0$, then $U_{0}(x)$ is non-singular for all $x \varepsilon J$ satisfying $\left|x-x_{0}\right| \leqq \frac{1}{2} \delta$. Now suppose that $(c, d)$ is an open subinterval of $J$ of length not exceeding $\delta$ and containing $n+l(l \geqq 1)$ focal points $x_{1} \leqq x_{2} \leqq \cdots \leqq$ $x_{n+l}$ of a conjoined family $F$ of solutions of $(2.1)$ with base $(U(x) ; V(x))$, where each focal point is counted a number of times equal to its order. Then there exist $n+l$ solutions $u_{j}(x), v_{i}(x)$ of $(2.1)$ of the form $u_{i}(x)=U(x) \xi_{i}, v_{i}(x)=$ $V(x) \xi_{i}$ such that $u_{i}\left(x_{i}\right)=0$, and if $\eta_{i}(x)=u_{i}(x)$ on $\left[c, x_{i}\right], \eta_{j}(x) \equiv 0$ on $\left[x_{i}, d\right]$, then $\eta_{1}(x), \cdots, \eta_{n+l}(x)$ are linearly independent on $[c, d]$. One may verify directly that $I\left(\eta_{j}, \eta_{k} ; c, d\right)=-\eta_{j}^{*}(c) V(c) \xi_{k}(j, k=1, \cdots, n+l)$, and consequently, for constants $d_{1}, \cdots, d_{n+l}$ not all zero and such that $\eta(x)=\eta_{1}(x) d_{1}+\cdots+$ $\eta_{n+l}(x) d_{n+l}$ satisfies $\eta(c)=0$, we have

$I(\eta ; c, d)=\sum_{j, k=1}^{n+l} \bar{d}_{j} d_{k} I\left(\eta_{j}, \eta_{k} ; c, d\right)=-\eta^{*}(c) V(c)\left(\xi_{1} d_{1}+\cdots+\xi_{n+l} d_{n+l}\right)=0$.

On the other hand, the first conclusion of the lemma and Theorem 3.1 implies the contradictory result $I(\eta ; c, d)>0$, so that $F$ can have at most $n$ focal points on a subinterval $(c, d)$ of length not exceeding $\delta$. This latter result clearly implies that the focal points of a conjoined family $F$ are isolated. 
If $J: a \leqq x \leqq b$ is a subinterval of $J_{0}$ and $x_{1}, \cdots, x_{m}$ are values such that $a=x_{0}<x_{1}<\cdots<x_{m}<x_{m+1}=b$ and (2.1) is non-oscillatory on each subinterval $\left[x_{i-1}, x_{i}\right](j=1, \cdots, m+1)$, then, for arbitrary $n$-dimensional vectors $z_{j}=\left(z_{\alpha i}\right)(\alpha=1, \cdots, n ; j=0,1, \cdots, m)$, there is a unique solution $u=$ $u_{z, j}(x), v=v_{z, j}(x)$ of $(2.1)$ such that

$$
u_{z, j}\left(x_{i-1}\right)=z_{j-1}, \quad u_{z, i}\left(x_{i}\right)=z_{i} \quad(j=1, \cdots, m+1),
$$

with the understanding that $z_{m+1}=0$. The corresponding vector function

$$
u_{z}(x)=u_{z, i}(x), \quad x_{i-1} \leqq x \leqq x_{i} \quad(j=1, \cdots, m+1),
$$

is then continuous on $J$ and linear in the components of the $n(m+1)$-dimensional vector

$$
\begin{aligned}
& \quad z=\left(z^{(p)}\right) \quad(p=1, \cdots, n m+n) \\
& \text { with } \quad z^{(n j+\alpha)}=z_{\alpha i} \quad(\alpha=1, \cdots, n ; j=0,1, \cdots, m) .
\end{aligned}
$$

If $G$ is an $n \times n$ hermitian matrix it follows from the hermitian nature of the integral $I\left(\eta_{1}, \eta_{2} ; a, b\right)$ that for $z, z^{\prime}$ two $n(m+1)$-dimensional vectors of the form (4.3) we have

$$
H\left(z^{\prime}, z\right) \equiv z_{0}^{\prime *} G z_{0}+I\left(u_{z^{\prime}}, u_{z} ; a, b\right)=z^{\prime *} H z,
$$

where $H$ is an $n(m+1)$-dimensional hermitian matrix. As usual, we set $H(z)=$ $H(z, z)$.

For Monse's treatment of focal points the result of the following theorem is basic. A proof of this result will be given here, since the usual proof of the result for differential systems employs certain continuity properties of solutions that do not hold for the general system (2.1) (see, for example, Monse [7; Ch. III, §3]).

Theorem 4.1. If $J: a \leqq x \leqq b$ is a subinterval of $J_{0}, G$ is an $n \times n$ hermitian matrix, while $x_{1}, \cdots, x_{m}$ and $u_{z}(x)$ are specified as above, then $H$ is of rank $n(m+1)-r$ if and only if $x=b$ is a focal point of order $r$ of the conjoined family of solutions $F$ with base $(U(x) ; V(x))$ determined by $U(a)=E, V(a)=G$. $M o r e o v e r$, the elements of $H$ are continuous functions of the elements of $G$ and the values $x_{1}, x_{2}, \cdots, x_{m}, x_{m+1}=b$.

In terms of the solutions $u=u_{z, i}(x), v=v_{z, i}(x)$ determined by (4.1) we have that

$$
H\left(z^{\prime}, z\right)=z_{0}^{\prime *}\left[G z_{0}-v_{z, 1}(a)\right]+\sum_{i=1}^{m} z_{j}^{\prime *}\left[v_{z, i}\left(x_{i}\right)-v_{z, j+1}\left(x_{i}\right)\right] .
$$

Since $H z=0$ if and only if $H\left(z^{\prime}, z\right)=0$ for arbitrary $z^{\prime}$, it follows that $H$ is of rank $n(m+1)-r$ if and only if there are $r$ linearly independent vectors $z$ such that $v_{z, 1}(a)=G z_{0}, v_{z, i}\left(x_{i}\right)=v_{z, j+1}\left(x_{i}\right)(j=1, \cdots, m)$; which is clearly equivalent to the condition that there are exactly $r$ linearly independent solutions $u(x), v(x)$ of (2.1) such that $v(a)=G u(a), u(b)=0$, that is, $x=b$ is a focal point 
of order $r$ for the conjoined family with base $(U(x) ; V(x))$ determined by $U(a)=$ $E, V(a)=G$.

The statement on the continuity of the elements of $H$ is clearly equivalent to the condition that for arbitrary fixed $z, z^{\prime}$ the quantity $H\left(z^{\prime}, z\right)$ is a continuous function of the elements of $G$ and the values $x_{1}, x_{2}, \cdots, x_{m}, x_{m+1}=b$. As $u_{z^{\prime}, j}\left(x_{i}\right)=u_{z^{\prime}, j+1}\left(x_{i}\right)=z_{j}^{\prime}(j=1, \cdots, m)$, expression (4.5) may be written

$$
H\left(z^{\prime}, z\right)=z_{0}^{\prime *}\left[G z_{0}-v_{z, 1}(a)\right]+\sum_{j=1}^{m}\left[u_{z^{\prime}, j+1}^{*}\left(x_{j}\right) v_{z, j}\left(x_{j}\right)-u_{z^{\prime}, j}^{*}\left(x_{j}\right) v_{z, j+1}\left(x_{j}\right)\right] .
$$

Now if $u_{\beta}(x), v_{\beta}(x)(\beta=1, \cdots, 2 n)$ are linearly independent solutions of $(2.1)$ on $J$, then for $j=1, \cdots, m+1$ there exist constants $c_{\beta, j}(\beta=1, \cdots, 2 n ; j=$ $1, \cdots, m+1)$ such that

$$
u_{z, j}(x)=\sum_{\beta=1}^{2 n} u_{\beta}(x) c_{\beta, j}, \quad v_{z, j}(x)=\sum_{\beta=1}^{2 n} v_{\beta}(x) c_{\beta, i}
$$

where $c_{\beta}=c_{\beta, j}(\beta=1, \cdots, 2 n)$ is the unique solution of the linear equations

$$
\sum_{\beta=1}^{2 n} u_{\beta}\left(x_{i-1}\right) c_{\beta}=z_{j-1}, \quad \sum_{\beta=1}^{2 n} u_{\beta}\left(x_{j}\right) c_{\beta}=z_{i}
$$

with $z_{m+1}=0$. Let $c_{\beta, j}^{\prime}(\beta=1, \cdots, 2 n ; j=1, \cdots, m+1)$ be the corresponding constants for $u_{z^{\prime}, j}(x), v_{z^{\prime}, j}(x)$ expressed in terms of the $u_{\beta}(x), v_{\beta}(x)$. In view of the continuity of the vector functions $u_{\beta}(x)$, it follows that the $c_{\beta, j}$ and $c_{\beta, j}^{\prime}$ are continuous functions of $x_{1}, \cdots, x_{m}, x_{m+1}=b$ as long as these values are on a range with (2.1) non-oscillatory on each subinterval $\left[x_{i-1}, x_{j}\right](j=1, \cdots$, $m+1)$. Consequently the values $u_{z^{\prime}, j+1}\left(x_{i}\right)$ and $u_{z^{\prime}, j}\left(x_{j}\right)$ in (4.6) are continuous in $x_{1}, \cdots, x_{m}, x_{m+1}=b$. On the other hand, since the $v_{\beta}(x)$ in general are not continuous, the desired continuity property is not immediate from (4.6). However, upon interchanging the roles of $z, z^{\prime}$ in (4.6) we obtain also

$$
H\left(z^{\prime}, z\right)=\left[z_{0}^{\prime *} G-v_{z^{\prime}, 1}^{*}(a)\right] z_{0}+\sum_{j=1}^{m}\left[v_{z^{\prime}, j}^{*}\left(x_{j}\right) u_{z, j+1}\left(x_{j}\right)-v_{z^{\prime}, j+1}^{*}\left(x_{i}\right) u_{z, j}\left(x_{j}\right)\right],
$$

and addition of the two expressions (4.6), (4.7) yields

$$
\begin{aligned}
2 H\left(z^{\prime}, z\right)=z_{0}^{\prime *}\left[G z_{0}-v_{z, 1}(a)\right]+\left[z_{0}^{\prime *} G\right. & \left.-v_{z^{\prime}, 1}^{*}(a)\right] z_{0} \\
& +\sum_{j=1}^{m} \sum_{\alpha, \beta=1}^{2 n}\left(\bar{c}_{\alpha, j+1}^{\prime} D_{\alpha \beta} c_{\beta, j}-\bar{c}_{\alpha, j}^{\prime} D_{\alpha \beta} c_{\beta, j+1}\right),
\end{aligned}
$$

where $D_{\alpha \beta}(\alpha, \beta=1, \cdots, 2 n)$ are the constant values $\left\{u_{\alpha}, u_{\beta}\right\} \equiv u_{\alpha}^{*}(x) v_{\beta}(x)-$ $v_{\alpha}^{*}(x) u_{\beta}(x)$. In view of this expression for $H\left(z^{\prime}, z\right)$, the stated continuity property is a direct consequence of the continuity of the $c_{\beta, j}, c_{\beta, j}^{\prime}$.

With the aid of Theorem 4.1 the method of Monse ([6, §7] or [7; Ch. III, §6]) yields the result that if $x=b$ is not a focal point of the conjoined family $F$ with base $(U(x) ; V(x))$ determined by $U(a)=E, V(a)=G$, then the negative type number of $H(z)$ is equal to the number of focal points of this family between $x=a$ and $z=b$, with focal points counted according to their orders. 
Suppose now that $J: a \leqq x \leqq b$ is a subinterval of $J_{0}$, the values $x_{1}, \cdots, x_{m}$ are as above, and $u_{z}(x)$ is the continuous vector function (4.2) given in terms of the solutions $u=u_{z, i}(x), v=v_{z, i}(x)$ specified by (4.1), where now $z_{0}=0=$ $z_{m+1}$. Then $u_{z}(x)$ is linear in the components of the $n m$-dimensional vector

$$
\begin{aligned}
& \zeta=\left(\zeta^{(\alpha)}\right) \quad(q=1, \cdots, n m) \\
& \text { with } \quad \zeta^{(i n-n+\alpha)}=z_{\alpha, j} \quad(\alpha=1, \cdots, n ; j=1, \cdots, m),
\end{aligned}
$$

and for two such vectors $\zeta, \zeta^{\prime}$ we have

$$
Q\left(\zeta^{\prime}, \zeta\right) \equiv I\left(u_{z^{\prime}}, u_{z} ; a, b\right)=\zeta^{*} Q \zeta
$$

where $Q$ is an $n m$-dimensional hermitian matrix. Analogous to Theorem 4.1, one may now establish that the elements of $Q$ are continuous functions of $a, x_{1}, \cdots$, $x_{m}, b$. By the method of Morse it then follows that $x=b$ is a conjugate point of $x=a$ of order $r$ if and only if the rank of $Q$ is $n m-r$, and that if $x=b$ is not conjugate to $x=a$ then the negative type number of $Q(\zeta) \equiv Q(\zeta, \zeta)$ is equal to the sum of the orders of the conjugate points of $x=a$ on $a<x<b$, and also equal to the sum of the orders of the conjugate points of $x=b$ on this interval. Once these results are obtained, one may proceed by Monse's method (see [6; §9]) to show that for $a \varepsilon J_{0}$ the $k^{\text {th }}$ right-hand conjugate point of $x=a$ advances or regresses continuously with $x=a$, as long as it remains on $J_{0}$. For the general problem under consideration here one may also establish by MonsE's method results that are direct extensions of Theorem 6 , with its corollaries, and Theorems $7,9,10$ of Morse [6].

The negative type numbers of the above hermitian forms $H(z), Q(\zeta)$ may be given also in terms of the number of negative proper values of associated boundary value problems. Prefatory to the statement of specific results of this nature, we shall establish the following lemma.

Lemma 4.2. If $J: a \leqq x \leqq b$ is a subinterval of $J_{0}$ and $\eta(x) \varepsilon \Gamma(a, b)$, then throughout $J$

$$
|\eta(x)|^{2} \leqq \int_{a}^{b}\left(k \eta^{*} R \eta^{\prime}+k^{-1} \eta^{*} R^{-1} \eta+2(b-a)^{-1} \eta^{*} \eta\right) d x
$$

where $k$ is an arbitrary positive constant. has

If $\eta(x) \varepsilon \Gamma(a, b)$, then for $\frac{1}{2}(a+b) \leqq c \leqq b$ and $\theta(x)=(x-a) /(c-a)$ one

$$
|\eta(c)|^{2}=\int_{a}^{c}\left[|\eta|^{2} /(c-a)+\left(\eta^{*} \eta^{\prime}+\eta^{*} \eta\right) \theta\right] d x .
$$

As $0 \leqq \theta(x) \leqq 1$ on $[a, c], 1 /(c-a) \leqq 2 /(b-a)$, and

$$
\left|\eta^{*} \eta^{\prime}+\eta^{*} \eta\right| \leqq k \eta^{*} R \eta^{\prime}+k^{-1} \eta^{*} R^{-1} \eta
$$

for arbitrary $k>0$, inequality (4.8) is seen to hold for $\frac{1}{2}(a+b) \leqq x \leqq b$. An analogous argument shows that this inequality holds for $a \leqq x \leqq \frac{1}{2}(a+b)$. 
Now let $\phi(x)$ be a positive function which is integrable on arbitrary compact subintervals of $J_{0}$, and such that for a given subinterval $J: a \leqq x \leqq b$ of $J_{0}$ there are values $c_{1}=c_{1}(J), c_{2}=c_{2}(J)$ such that

$$
\phi(x) E-\left[c_{1} R^{-1}(x)+c_{2} E\right] \geqq 0 \text { for } x \varepsilon J .
$$

If $\eta(x) \varepsilon \Gamma(a, b)$ then

$$
\int_{a}^{b} \eta^{*}(x)[d M(x)] \eta(x) \geqq-[h(b)-h(a)]\left|\eta\left(x^{*}\right)\right|^{2},
$$

where $h(x)$ is as in the proof of Theorem 2.1 and $x=x^{*}$ is a value at which $|\eta(x)|$ assumes its maximum on $J$. Consequently, if $G$ is an $n \times n$ hermitian matrix it follows from Lemma 4.2 that for a given subinterval $J: a \leqq x \leqq b$ there exists a value $\lambda_{*}<0$ such that, for $\lambda<\lambda_{*}$ and arbitrary $\eta(x) \varepsilon \Gamma(a, b)$,

$$
\eta^{*}(a) G \eta(a)+\int_{a}^{b}\left(\eta^{\prime *} R \eta^{\prime}-\lambda \phi(x) \eta^{*} \eta\right) d x+\int_{a}^{b} \eta^{*}(x)[d M(x)] \eta(x) \geqq 0 .
$$

Now let $J: a \leqq x \leqq b$ be a subinterval of $J_{0}$ such that $x=b$ is not a focal point of the conjoined family with base $(U(x) ; V(x))$ determined by $U(a)=E$, $V(a)=G$. If $a=x_{0}<x_{1}<\cdots<x_{m}<x_{m+1}=b$ and (2.1) is non-oscillatory on each subinterval $\left[x_{i-1}, x_{i}\right](j=1, \cdots, m+1)$, in view of the monotoneity of the left-hand member of (4.10) as a function of $\lambda$, it follows from Theorem 3.1 that for $\lambda<0$ the system

$$
R(x) u^{\prime}(x)=v(x), \quad d v(x)=\left[d\left(M(x)-\lambda E \int_{a}^{x} \phi(s) d s\right)\right] u(x)
$$

is also non-oscillatory on each $\left[x_{i-1}, x_{i}\right]$. If $H(z ; \lambda)$ denotes the corresponding hermitian form (4.4) for the functional appearing as the left-hand member of (4.10), it then follows that for $\lambda<\lambda_{*}$ the negative type number of $H(z ; \lambda)$ is zero. Moreover, from the expression for $H(z ; \lambda)$ corresponding to (4.7) and from Theorem 2.2, it follows that for fixed values of $a=x_{0}, x_{1}, \cdots, x_{m}, x_{m+1}=b$ the coefficients of $H(z ; \lambda)$ are continuous functions of $\lambda$ on $\lambda \leqq 0$. By the argument used by Morse [7; Ch. III, §2] one is then led to the following result.

Theorem 4.2. If $J: a \leqq x \leqq b$ is a subinterval of $J_{0}$ such that $x=b$ is not $a$ focal point of the conjoined family with base $(U(x) ; V(x))$ determined by $U(a)=E$, $V(a)=G$, and if $\phi(x)$ is a positive integrable function on $J$ satisfying (4.9), then the negative type number of the hermitian form $H(z)$ is equal to the number of negative proper values of the boundary problem consisting of the generalized differential system (4.11) and the two-point conditions

$$
G u(a)-v(a)=0, \quad u(b)=0,
$$

each proper value being counted according to its index.

Correspondingly, if $J: a \leqq x \leqq b$ is a subinterval of $J_{0}$ such that $x=b$ is not conjugate to $x=a$ and if $\phi(x)$ is as above, then the negative type number of 
the hermitian form $Q(\zeta)$ is equal to the number of negative proper values of the boundary problem consisting of (4.11) and the two-point conditions $u(a)=0=$ $u(b)$.

Results analogous to those of $\$ \S 3-7$ in Ch. IV of Morse [7] may be established for self-adjoint boundary problems involving a generalized differential system (2.12) with coefficient matrices satisfying hypothesis $\left(\mathrm{H}_{\lambda}\right)$ on arbitrary compact subintervals $J, \lambda_{1} \leqq \lambda \leqq \lambda_{2}$ of the ranges $J_{0}, J_{\lambda}$ of $x$ and $\lambda$, respectively, with $R(x, \lambda)>0$ a.e. on $J_{0}$ for fixed $\lambda \varepsilon J_{\lambda}$, and for which certain associated hermitian functionals possess suitable monotoneity properties as functions of $\lambda$.

It is to be remarked that the extension to systems (2.1) of the Sturmian theory may be attained also by the general method of natural isoperimetric conditions employed by BIRKHoFf \& Hestenes [1] in the more classical problem, as well as by the functional method of Hestenes [5].

5. Principal solutions. We shall consider now a self-adjoint system (2.1) whose coefficients matrices satisfy hypothesis $(\mathrm{H})$ on arbitrary compact subintervals of $J_{0}: a<x<\infty$. If $(U(x) ; V(x))$ is a solution of (3.6) with $U(x)$ non-singular on a given subinterval $X_{0}$ of $J_{0}$ and if $K$ is the $n \times n$ constant matrix such that $\{U, U\} \equiv K$, then, corresponding to the results at the beginning of Section 3 of REID [10], one may show that a $2 n \times r$ matrix $\left(U_{0}(x) ; V_{0}(x)\right)$ is a solution of (3.6) with $\left\{U, U_{0}\right\} \equiv K_{0}$ if and only if

$$
U_{0}(x)=U(x) H(x), \quad V_{0}(x)=V(x) H(x)+U^{*-1}(x)\left[K_{0}-K H(x)\right],
$$

where $H(x)$ is an $n \times r$ matrix which is a solution of

$$
H^{\prime}(x)=U^{-1}(x) R^{-1}(x) U^{*-1}(x)\left[K_{0}-K H(x)\right], \quad x \varepsilon X_{0} ;
$$

that is, $H(x)$ is a.c. and (5.2) holds a.e. on $X_{0}$. Moreover, the general solution of $(5.2)$ is

$$
H(x)=T(x, s ; U)\left[H_{0}+S(x, s ; U) K_{0}\right],
$$

where $T(x)=T(x, s ; U)$ is the solution of the matrix differential system

$$
T^{\prime}=-U^{-1}(x) R^{-1}(x) U^{*-1}(x) K T, \quad T(s)=E,
$$

$H_{0}$ is a constant matrix, and for $x, s \varepsilon X_{0}$

$$
S(x, s ; U)=\int_{s}^{x} T^{-1}(t, s ; U) U^{-1}(t) R^{-1}(t) U^{*^{-1}}(t) d t .
$$

Corresponding to the definition of REID [10], a $2 n \times n$ matrix solution $(U(x) ; V(x))$ of (3.6) is termed a principal solution (at $\infty$ ) if $U(x)$ is non-singular on a subinterval $X_{U}: a_{U} \leqq x<\infty$ of $J_{0}$, and $S^{-1}(x, s ; U) \rightarrow 0$ as $x \rightarrow \infty$ for at least one (and consequently all) $s \varepsilon X_{U}$. All of the results of $\$ \S 3-6$ of REID [10] may be extended to the general system under consideration here, with only the most minor adjustments in details of proof. We shall therefore limit our discussion here to the following brief statements. 
If (2.1) is a self-adjoint system which is non-oscillatory on $J_{0}: a<x<\infty$ and $R(x)>0$ a.e. on this interval, then, as in $\$ \$ 5,6$ of ReID [10], one may show that if $(U(x) ; V(x))$ is a solution of (3.6) with $U(x)$ non-singular on a subinterval $X_{U}: a_{U} \leqq x<\infty$ of $J_{0}$, then for $s \varepsilon X_{U}$ the matrix $M(s ; U)=\lim _{t \rightarrow \infty} S^{-1}(t, s ; U)$ exists and is finite. Moreover, $M(s ; U)=0$ and $(U(x) ; V(x))$ is a principal solution of (3.6) if and only if $U(x)=U_{r, \infty}(x) C, V(x)=V_{r, \infty}(x) C$, with $r$ any fixed value on $J_{0}, C$ a non-singular constant matrix, and $\left(U_{r, \infty}(x) ; V_{r, \infty}(x)\right)$ the matrix of conjoined solutions determined by $U_{r, \infty}(x)=\lim _{s \rightarrow \infty} U_{r, s}(x), V_{r, \infty}(x)=$ $\lim _{s \rightarrow \infty} V_{r, s}(x)$, where $\left(U_{r, s}(x) ; V_{r, s}(x)\right)$ is the solution of (3.6) satisfying $U_{r, s}(r)=E, U_{r, s}(s)=0$ for $r<s<\infty$. Corresponding to certain results of the corollary to Theorem 6.1 and Theorem 6.2 of Rerd [10], one has that if $(U(x) ; V(x))$ is a principal solution of $(3.6)$, then for a solution $\left(U_{0}(x) ; V_{0}(x)\right)$ of this system the matrix $\left\{U_{0}, U\right\}$ is non-singular if and only if $U_{0}(x)$ is nonsingular for large $x$ and $U_{0}^{-1}(x) U(x) \rightarrow 0$ as $x \rightarrow \infty$; moreover, if $\left\{U_{0}, U\right\}$ is non-singular, then for $s$ sufficiently large the matrix $S\left(\infty, s ; U_{0}\right)=\lim _{t \rightarrow \infty}$ $S\left(t, s ; U_{0}\right)$ exists and is non-singular and

$$
U(s)=-U_{0}(s) S\left(\infty, s ; U_{0}\right)\left\{U_{0}, U\right\} .
$$

It is to be remarked that for the real scalar generalized second order differential operators considered by FeLLer [2], the solutions that he has termed "minimal" at the individual end-points (see [2; §3]) are "principal" in the sense of the present paper.

6. Systems of difference equations. Now suppose that the $n \times n$ matrices $R(x), M(x)$ satisfy hypothesis $(\mathrm{H})$ on an interval $J: a \leqq x \leqq b$ and that there exist values $t_{1}, \cdots, t_{m}(m \geqq 1)$ with $a=t_{0}<t_{1}<\cdots<t_{m}<t_{m+1}=b$ and constant matrices $R_{i}, M_{i}$ such that

$$
R(x) \equiv R_{i}, \quad M(x) \equiv M_{j} \quad \text { on } \quad t_{j}<x<t_{i+1} \quad(j=0,1, \cdots, m) .
$$

If $u(x), v(x)$ is a solution of (2.1) on $J$, then on this interval $u(x)$ is a continuous vector function that is linear on each subinterval $\left[t_{i}, t_{i+1}\right]$, and for

$$
\begin{gathered}
G_{a+1}=M_{a+1}-M_{a} \quad(q=0,1, \cdots, m-1), \\
\Delta u\left(t_{j}\right)=u\left(t_{j+1}\right)-u\left(t_{j}\right), \quad \Delta t_{j}=t_{j+1}-t_{j} \quad(j=0,1, \cdots, m)
\end{gathered}
$$

the sequence $u\left(t_{0}\right), u\left(t_{1}\right), \cdots, u\left(t_{m+1}\right)$ satisfies the system of difference equations

$$
R_{a+1} \frac{\Delta u\left(t_{q+1}\right)}{\Delta t_{q+1}}-R_{q} \frac{\Delta u\left(t_{q}\right)}{\Delta t_{q}}-G_{q+1} u\left(t_{q+1}\right)=0 \quad(q=0,1, \cdots, m-1) .
$$

Conversely, if on $J$ the vector function $u(x)$ is continuous, linear on each subinterval $\left[t_{j}, t_{i+1}\right]$, and satisfies (6.4), then a solution of (2.1) is given by $u=u(x)$, $v=v(x)$ with 


$$
\begin{aligned}
v\left(t_{0}\right) & =R_{0} \frac{\Delta u\left(t_{0}\right)}{\Delta t_{0}}+\left[M\left(t_{0}\right)-M_{0}\right] u\left(t_{0 j},\right. \\
v(x) & =R_{i} \frac{\Delta u\left(t_{j}\right)}{\Delta t_{i}}, \quad t_{i}<x<t_{i+1}, \\
v\left(t_{i+1}\right) & =R_{i} \frac{\Delta u\left(t_{i}\right)}{\Delta t_{i}}+\left[M\left(t_{i+1}\right)-M_{i}\right] u\left(t_{i+1}\right) \quad(j=0,1, \cdots, m) .
\end{aligned}
$$

If $R(x), M(x)$ are hermitian on $J$ and $R_{j}>0(j=0,1, \cdots, m)$, then the hypotheses employed in Sections 3,4 are satisfied, and the results of these sections have as specializations results for the systems of difference equations (6.4). In particular, the statement that for two solutions $u_{1}(x), v_{1}(x)$ and $u_{2}(x)$, $v_{2}(x)$ the quantity $\left\{u_{1}, u_{2}\right\}=u_{1}^{*}(x) v_{2}(x)-v_{1}^{*}(x) u_{2}(x)$ is equal to a constant $c$ is equivalent to the condition that

$$
u_{1}^{*}\left(t_{j}\right) R_{i} \frac{\Delta u_{2}\left(t_{j}\right)}{\Delta t_{j}}-\frac{\Delta u_{1}^{*}\left(t_{j}\right)}{\Delta t_{j}} R_{i} u_{2}\left(t_{j}\right)=c \quad(j=0,1, \cdots, m) .
$$

A direct treatment of real self-adjoint systems (6.4), with the derivation of certain central oscillation and comparison theorems, has been presented in the dissertation of V. C. HARRIs [4]. The above consideration of a general self-adjoint system (2.1) that includes as individual special cases differential systems and difference systems highlights the common aspects of these two instances and provides a better understanding of the intimate relationships that have persisted in the historical development of the theory of real scalar linear differential equations of the second order and corresponding difference equations (see, for example, FonT [3; Chapter X]).

7. Related generalized differential systems. In view of the general form of the second variation for a simple integral variational problem, there will be considered now a more general system that may be reduced to the form (2.1). Suppose that $R(x), Q_{1}(x), Q_{2}(x), P(x)$, and $M(x)$ are $n \times n$ matrices satisfying on $J: a \leqq x \leqq b$ the following hypothesis:

$\left(\mathrm{H}_{0}\right) R(x)$ is non-singular with $R(x)$ and $R^{-1}(x)$ integrable, $Q_{1}(x)$ and $Q_{2}(x)$ are measurable and essentially bounded, $P(x)$ is integrable, and $M(x)$ is of bounded variation.

By a solution $u(x), v(x)$ of the system

$$
\begin{gathered}
R(x) u^{\prime}(x)+Q_{1}(x) u(x)=v(x), \\
d v(x)=\left[Q_{2}(x) u^{\prime}(x)+P(x) u(x)\right] d x+[d M(x)] u(x)
\end{gathered}
$$

on $J$ will be meant an a.c. $u(x)$ and an associated $v(x)$ of b.v. on $J$ such that

$$
v(x)=\int_{a}^{x}\left[Q_{2}(s) u^{\prime}(s)+P(s) u(s)\right] d s+\int_{a}^{x}[d M(s)] u(s)+v(a), \quad x \varepsilon J
$$


and $R u^{\prime}+Q_{1} u=v$ a.e. on $J$. Corresponding to (2.14), let

$$
\begin{aligned}
I_{0}\left(\eta_{1}, \eta_{2} ; a, b\right)=\int_{a}^{b} & {\left[\eta_{1}^{\prime *}\left(R \eta_{2}^{\prime}+Q_{1} \eta_{2}\right)\right.} \\
& \left.\quad+\eta_{1}^{*}\left(Q_{2} \eta_{2}^{\prime}+P \eta_{2}\right)\right] d x+\int_{a}^{b} \eta_{1}^{*}(x)[d M(x)] \eta_{2}(x) .
\end{aligned}
$$

In a manner completely analogous to the proof of Lemma 2.1 one may establish the following result.

Lemma 7.1. For a given vector function $u(x)$ there is an associated $v(x)$ such that $u(x), v(x)$ is a solution of (7.1) on $a \leqq x \leqq b$ if and only if on this interval $u(x)$ is a.c., $R(x) u^{\prime}(x)+Q_{1}(x) u(x)$ is essentially bounded, and $I_{0}(\eta, u ; a, b)=0$ for arbitrary a.c. $n$-dimensional vector functions $\eta(x)$ satisfying $\eta(a)=0=\eta(b)$.

Let $G(x)$ be a fundamental matrix solution of

$$
G^{\prime}(x)+R^{-1}(x) Q_{1}(x) G(x)=0,
$$

that is, $G(x)$ is a non-singular a.c. matrix such that (7.3) holds a.e. on $J$. Correspondingly, let $H(x)$ be a fundamental matrix solution of

$$
H^{\prime}(x)+R^{*-1}(x) Q_{2}^{*}(x) H(x)=0 .
$$

Under hypothesis $\left(\mathrm{H}_{0}\right)$ the matrices $R_{0}(x), P_{0}(x)$ defined as

$$
R_{0}=H^{*} R G, \quad P_{0}=H^{*}\left(P-Q_{2} R^{-1} Q_{1}\right) G
$$

are such that $R_{0}(x)$ is non-singular, and $R_{0}(x), R_{0}^{-1}(x), P_{0}(x)$ are integrable on $J$, while the corresponding matrix

$$
M_{0}(x)=\int_{a}^{x} H^{*}(s)[d M(s)] G(s)+\int_{a}^{x} P_{0}(s) d s
$$

is of b.v. on this interval. The theory of a system (7.1) is then reduced to the theory of a system (2.1), by the following result.

Theorem 7.1. If the coefficient matrices of (7.1) satisfy $\left(\mathrm{H}_{0}\right)$ on $J: a \leqq x \leqq b$ and $G(x), H(x)$ are fundamental matrix solutions of the respective equations (7.4), (7.5), then $u(x), v(x)$ is a solution of (7.1) on $J$ if and only if

$$
u_{0}(x)=G^{-1}(x) u(x), \quad v_{0}(x)=H^{*}(x) v(x)
$$

is a solution on this interval of

$$
R_{0}(x) u_{0}^{\prime}(x)=v_{0}(x), \quad d v_{0}(x)=\left[d M_{0}(x)\right] u_{0}(x),
$$

where $R_{0}(x), M_{0}(x)$ are defined by (7.6), (7.7).

If $u(x), u_{0}(x)$ are vector functions satisfying $u(x)=G(x) u_{0}(x)$ on $J$, then clearly $u(x)$ is a.c. if and only if $u_{0}(x)$ is a.c.; moreover, $R u^{\prime}+Q_{1} u=R G u_{0}^{\prime}$ a.e., so that $R u^{\prime}+Q_{1} u$ is essentially bounded on $J$ if and only if $R_{0} u_{0}^{\prime}=H^{*} R G u_{0}^{\prime}$ 
is essentially bounded on this interval. Correspondingly, if $\eta(x)=H(x) \eta_{0}(x)$ on $J$ then $\eta(x)$ is a.c. and satisfies $\eta(a)=0=\eta(b)$ if and only if $\eta_{0}(x)$ is a.c. and satisfies $\eta_{0}(a)=0=\eta_{0}(b)$. Therefore, if $\eta(x)$ and $u(x)$ are a.c. on $J$ and $R u^{\prime}+Q_{1} u$ is essentially bounded on this interval, then $u_{0}(x)=G^{-1}(x) u(x)$, $\eta_{0}(x)=H^{-1}(x) \eta(x)$ are such that

$$
\int_{a}^{b} \eta^{*}(x)[d M(x)] u(x)=\int_{a}^{b} \eta_{0}^{*}(x)\left[d\left(\int_{a}^{x} H^{*}(s)[d M(s)] G(s)\right)\right] u_{0}(x),
$$

and

$$
I_{0}(\eta, u ; a, b)=\int_{a}^{b} \eta_{0}^{* *}(x) R_{0}(x) u_{0}^{\prime}(x) d x+\int_{a}^{b} \eta_{0}^{*}(x)\left[d M_{0}(x)\right] u_{0}(x) .
$$

In view of Lemmas 2.1 and 7.1 it follows that if $u(x)$ is a.c. and $R u^{\prime}+Q_{1} u$ is essentially bounded on $J$, then there is a $v(x)$ such that $u(x), v(x)$ is a solution of (7.1) if and only if there is a $v_{0}(x)$ such that $u_{0}(x)=G^{-1}(x) u(x), v_{0}(x)$ is a solution of (7.9). Now when such vector functions $v(x), v_{0}(x)$ exist we have $R_{0}(x) u_{0}^{\prime}(x)=v_{0}(x)$ and $R(x) u^{\prime}(x)+Q_{1}(x) u(x)=v(x)$ a.e. on $J$, so that $H^{*}(x) v(x)=v_{0}(x)$ a.e. on this interval. Moreover, since

$$
\begin{aligned}
H^{*}(x) v(x)= & \int_{a}^{x} H^{\prime *}(s) v(s) d s+\int_{a}^{x} H^{*}(s)[d v(s)]+H^{*}(a) v(a) \\
= & \int_{a}^{x}\left[H^{\prime *}\left(R u^{\prime}+Q_{1} u\right)+H^{*}\left(Q_{2} u^{\prime}+P u\right)\right] d s \\
& +\int_{a}^{x} H^{*}(s)[d M(s)] u(s)+H^{*}(a) v(a),
\end{aligned}
$$

it follows from (7.4), (7.5) that

$$
H^{*}(x) v(x)=v_{0}(x)-v_{0}(a)+H^{*}(a) v(a), \quad x \varepsilon J .
$$

That is, $H^{*}(x) v(x)-v_{0}(x)$ is constant on $J$, and since $H^{*}(x) v(x)-v_{0}(x)=0$ a.e. on this interval we have $H^{*}(x) v(x) \equiv v_{0}(x)$.

If $R(x) \equiv R^{*}(x), Q_{2}(x) \equiv Q_{1}^{*}(x), P(x) \equiv P^{*}(x)$, and $M(x) \equiv M^{*}(x)$, then one may choose $H(x) \equiv G(x)$ and $R_{0}(x), P_{0}(x), M_{0}(x)$ are hermitian on $J$. If $u_{\alpha}(x), v_{\alpha}(x)(\alpha=1,2)$ are solutions of (7.1) and $u_{0 \alpha}(x)=G^{-1}(x) u_{\alpha}(x), v_{0 \alpha}(x)=$ $G^{*}(x) v_{\alpha}(x)$ are the corresponding solutions of (7.9), then

$$
u_{1}^{*}(x) v_{2}(x)-v_{1}^{*}(x) u_{2}(x) \equiv u_{01}^{*}(x) v_{02}(x)-v_{01}^{*}(x) u_{02}(x) .
$$

In particular, if $F$ is a conjoined family of solutions of (2.1) with base $(U(x) ; V(x))$, then $\left(U_{0}(x) ; V_{0}(x)\right)$ with $U_{0}(x)=G^{-1}(x) U(x), V_{0}(x)=G^{*}(x) V(x)$ is the base of a conjoined family $F_{0}$ of solutions of (7.9) such that $x=x_{0}$ is a focal point of $F$ of order $r$ if and only if $x=x_{0}$ is a focal point of $F_{0}$ of order $r$. In consequence of these remarks, the results of $\$ \S 3,4,5$ for the system (7.9) admit ready interpretation as corresponding results for the system (7.1).

Finally, it is to be remarked that a system (2.1) on an interval $J: a \leqq x \leqq b$ 
is equivalent to a corresponding system

$$
R_{1}(x) u_{1}^{\prime}(x)=v_{1}(x), \quad d v_{1}(x)=\left[d M_{1}(x)\right] u_{1}(x)
$$

where the elements of $M_{1}(x)$ are step functions on $J$. Indeed, in view of the corollary to Theorem 2.1 and the remarks on the adjoint system (2.16) at the end of $\$ 2$, it follows that if $a=t_{0}<t_{1}<\cdots<t_{k}=b$ is a partition of $J$ of sufficiently small norm, then on each subinterval $\left[t_{i-1}, t_{j}\right]$ there is a solution $\left(U_{i}(x) ; V_{i}(x)\right)$ of $(3.6)$ and a solution $\left(Y_{i}(x) ; Z_{j}(x)\right)$ of the corresponding adjoint matrix system

$$
R^{*}(x) Y^{\prime}(x)=Z(x), \quad d Z(x)=\left[d M^{*}(x)\right] Y(x),
$$

with $U_{j}(x)$ and $Y_{j}(x)$ non-singular on $\left[t_{i-1}, t_{i}\right]$, while $Y_{i}^{*}(x) V_{i}(x)-Z_{i}^{*}(x) U_{i}(x)$ $\equiv 0$. In particular, for the points $t_{i}$ sufficiently close together these conditions are satisfied by the solutions of (3.6) and (7.11) specified by the initial conditions $U_{i}\left(t_{j-1}\right)=E, V_{j}\left(t_{i-1}\right)=0, Y_{i}\left(t_{i-1}\right)=E, Z_{i}\left(t_{i-1}\right)=0$. As the stated conditions are preserved when $\left(U_{i}(x) ; V_{i}(x)\right)$ is replaced by $\left(U_{i}(x) C_{i} ; V_{i}(x) C_{i}\right)$ and $\left(Y_{i}(x) ; Z_{j}(x)\right)$ is replaced by $\left(Y_{i}(x) D_{i} ; Z_{i}(x) D_{i}\right)$, where the $C_{i}, D_{j}$ are nonsingular constant matrices, without loss of generality we may assume that

$$
U_{j}\left(t_{j}\right)=U_{i+1}\left(t_{j}\right), \quad Y_{i}\left(t_{i}\right)=Y_{j+1}\left(t_{j}\right) \quad(j=1, \cdots, k-1),
$$

so that if

$$
U(x)=U_{j}(x), \quad Y(x)=Y_{j}(x), \quad t_{i-1} \leqq x \leqq t_{i} \quad(j=1, \cdots, k),
$$

then $U(x), Y(x)$ are a.c. and non-singular on $J$.

With $U(x), Y(x)$ thus defined, if $u(x), u_{1}(x)$ are vector functions related by $u(x)=U(x) u_{1}(x)$ on $J$ then $u(x)$ is a.c. and $R(x) u^{\prime}(x)$ is essentially bounded on $J$ if and only if $u_{1}(x)$ is a.c. and $R_{1}(x) u_{1}^{\prime}(x)$ is essentially bounded on this interval, where

$$
R_{1}(x)=Y^{*}(x) R(x) U(x)
$$

moreover, if $\eta(x)=Y(x) \eta_{1}(x)$ then $\eta(x)$ is a.c. and satisfies $\eta(a)=0=\eta(b)$ if and only if $\eta_{1}(x)$ is a.c. and satisfies $\eta_{1}(a)=0=\eta_{1}(b)$. Consequently, if $u(x)$ is a.c. with $R(x) u^{\prime}(x)$ essentially bounded on $J$, while $\eta(x)$ is a.c. and $\eta(a)=$ $0=\eta(b)$, corresponding to the transformation of Lemma 3.2 we have

$$
\begin{aligned}
I(\eta, u ; a, b) & =\int_{a}^{b} \eta_{1}^{\prime *}(x) R_{1}(x) u_{1}^{\prime}(x) d x+\sum_{i=1}^{k-1} \eta_{1}^{*}(x) Y^{*}\left(t_{j}\right)\left[V_{i}\left(t_{j}\right)-V_{i+1}\left(t_{j}\right)\right] u_{1}\left(t_{j}\right) \\
& =\int_{a}^{b} \eta_{1}^{\prime *}(x) R_{1}(x) u_{1}^{\prime}(x) d x+\int_{a}^{b} \eta_{1}^{*}(x)\left[d M_{1}(x)\right] u_{1}(x)
\end{aligned}
$$

where $M_{1}(x)$ is defined as

$$
M_{1}(a)=0, \quad M_{1}(x)=\sum_{a<t_{i}<x} Y^{*}\left(t_{j}\right)\left[V_{i}\left(t_{j}\right)-V_{i+1}\left(t_{j}\right)\right], \quad a<x \leqq b .
$$

From Lemma 2.1 it then follows that a vector function $u(x)$ belongs to a 
solution $u(x), v(x)$ of $(2.1)$ if and only if $u_{1}(x)=U^{-1}(x) u(x)$ belongs to a solution $u_{1}(x), v_{1}(x)$ of (7.10), where $R_{1}(x), M_{1}(x)$ are defined by (7.13), (7.14). In particular, if $(2.1)$ is self-adjoint then one may choose $\left(Y_{j}(x) ; Z_{i}(x)\right)=$ $\left(U_{j}(x) ; V_{j}(x)\right)$, in which case the matrices $R_{1}(x), M_{1}(x)$ are hermitian and (7.10) is self-adjoint.

It is to be remarked that although this transformation is of theoretical interest, its introduction would have provided little or no simplification of the treatment in $\$ \$ 3,4,5$.

\section{BibLIOGRAPHY}

[1] G. D. Birkhoff \& M. R. Hestenes, Natural isoperimetric conditions in the calculus of variations, Duke Math. J., 1 (1935), 198-286.

[2] W. Feller, Generalized second order differential operators and their lateral conditions, Illinois J. Math., 1 (1957), 459-504.

[3] T. ForT, Finite differences and difference equations in the real domain, Oxford, Clarendon Press, 1948.

[4] V. C. HARRIS, $A$ system of difference equations and an associated boundary value problem, Dissertation (Northwestern University), 1950.

[5] M. R. Hestenes, Applications of the theory of quadratic forms in Hilbert space to the calculus of variations, Pacific J. Math., 1 (1951), 525-581.

[6] M. Morse, $A$ generalization of the Sturm separation and comparison theorems in $n$-space, Math. Ann., 103 (1930), 72-91.

[7] M. Monse, The calculus of variations in the large, Amer. Math. Colloq. Publ. XVIII, 1934.

[8] W. T. ReID, Discontinuous solutions in the non-parametric problem of Mayer in the calculus of variations, Amer. J. Math., 57 (1935), 69-93.

[9] W. T. REID, Oscillation criteria for linear differential systems with complex coefficients, Pacific J. Math., 6 (1956), 733-751.

[10] W. T. REID, Principal solutions of non-oscillatory self-adjoint linear differential systems, Pacific J. Math., 8 (1958), 147-169.

[11] F. Riesz \& B. Sz.-NAGY, Functional analysis, F. Ungar Publishing Co., New York, 1955.

[12] B. Sz.-NAGY, Vibrations d'une corde non homogène, Bull. Soc. Math. France, 75 (1947), 193-209.

[13] D. V. WIDDER, The Laplace transform, Princeton Univ. Press, 1946.

Northwestern University

Evanston, Illinois 